\title{
On the Covering Law in Quantal Proposition Systems
}

\author{
WILHELM OCHS \\ Sektion Physik der Universität München
}

Received September 13, 1971

\begin{abstract}
A recent justification of the covering law by Jauch and Piron founded on the existence of ideal measurements of the first kind is shown to contain a gap. This gap is closed by an additional assumption about such measurements.
\end{abstract}

\section{$\S 1$}

In a recent paper [1], Jauch and Piron tried to found the atomicity and the validity of the covering law in the proposition systems of quantummechanical objects onto a new concept of state and on the existence of ideal measurements of the first kind. But their derivation of the covering law contains a gap. In the present paper we show at first by means of a counter-example that the assumptions of Jauch and Piron are not sufficient to derive the covering law. Thereupon we introduce an additional plausible assumption about ideal measurements of the first kind which enables - in conjunction with the assumptions of [1] - the establishment of the covering law.

Remark. As in the present paper we simply presuppose the atomicity of quantal proposition systems which Jauch and Piron justified on the basis of a new concept of state, we can evade the problematic hypotheses connected with this new state concept; consequently, our considerations are independent of these hypotheses, and they concern only the dependence of the covering law upon other more plausible properties of quantal proposition systems.

\section{$\S 2$}

We start by compiling the assumptions from which Jauch and Piron tried to derive the covering law. As the physical substantiation of these assumptions is not the subject of the present paper, we immediately combine a great many assumptions which are not at all trivial, to the following axiom:

Axiom I. In the set $L$ of the propositions of a quantum-mechanical system there exist a partial order relation $\leqq$ and an orthocomplementa- 
tion ' such that the proposition system $\mathscr{P}=\left(L, \leqq,^{\prime}\right)$ forms a complete, atomic, orthomodular lattice.

As for the definition of the several notions, its physical interpretation and for the justification of Axiom I, we refer to the literature [1-3].

In addition to Axiom I, Jauch and Piron suppose the existence of ideal measurements of the first kind. In order to understand the significance of this assumption for, and to be able to formulate it as a condition on $\mathscr{P}$, we need some preliminary reflection. In the Hilbert space formalism of conventional quantum mechanics, homogeneous states (pure cases) are represented by elementary projection operators. These elementary projection operators can be characterized, in the language of lattice theory, as atoms or, equivalently, as prime filters ${ }^{1}$. In this way the homogeneous quantum states (and only these) can be incorporated into the proposition calculus without the introduction of a notion of probability. Following Jauch and Piron we introduce:

Definition 1. By a pure state of a quantum system with the proposition system $\mathscr{P}$ we understand a prime filter of $\mathscr{P}^{2}$.

This definition characterizes a pure state by the set $S$ of all propositions (i.e. 0 -1-observables) the measurement of which yields the result 1 with certainty.

Notations. In accordance with [1], we use the term $x$ is true if a measurement of $x$ will give the result 1 with certainty; hence $x \in S$ means that $x$ is true in the state $S$. We denote the set of atoms of a lattice $\mathscr{V}$ by $\Omega(\mathscr{V})$ and the set of its prime filters by $\Pi(\mathscr{V})$.

From Axiom I and Definition 1 one immediately gets the following properties of pure states:

$$
\begin{gathered}
(\forall S) \mathbf{0} \notin S, \quad \mathbf{1} \in S, \\
x \in S, \quad x \leqq y \Rightarrow y \in S, \\
S_{1} \subset S_{2} \Rightarrow S_{1}=S_{2}, \\
x \in S, \quad y \in S \Rightarrow x \cap y \in S, \\
(\forall x \in \mathscr{P} \backslash\{\boldsymbol{0}\})(\exists S \in \Pi(\mathscr{P})) \quad x \in S,
\end{gathered}
$$

where $\mathbf{0}$ and $\mathbf{1}$ are the first and last element of $\mathscr{P}$ respectively.

${ }^{1}$ A principal filter of a lattice $\mathscr{V}$ is a subset $H$ of $\mathscr{V}$ of the form $H=\{x \in \mathscr{V} \mid b \leqq x\}$ for a certain element $b \in \mathscr{V}$. A principal filter $S$ of $\mathscr{V}$ is said to be a prime filter, if every principal filter $H$ with $S \subset H$ is either equal to $S$ or to $\mathscr{V}$.

${ }^{2}$ Definition 1 implies the existence of a bijection $\mathfrak{g}: \Omega(\mathscr{P}) \mapsto \Pi(\mathscr{P})$ of the set $\Omega(\mathscr{P})$ of all atoms of $\mathscr{P}$ onto the set $\Pi(\mathscr{P})$ of all pure states of the system, $\mathfrak{g}$ being uniquely defined by each of the equations

$$
\mathfrak{g}(x)=\{y \in \mathscr{P} \mid y \geqq x\}, \quad x \in S \Leftrightarrow x \geqq \mathfrak{g}^{-1}(S), \quad \Omega(\mathscr{P}) \cap S=\mathfrak{g}^{-1}(S) .
$$

In the following we use the more suggestive notation $S_{x}$ instead of $\mathrm{g}(x)$. 
In quantum mechanics [2], a measurement of a proposition $x$ is called of the first kind if the result 1 implies that $x$ is true immediately after the measurement. By virtue of the interpretation of $x^{\prime}$ it follows in particular that a measurement of the first kind of $x$ can not yield the result 1 if $x^{\prime}$ was true before the measurement. The measurement of a proposition $x$ is said to be ideal if every proposition compatible with $x$ and true before the measurement is still true after the measurement. In order to see the consequences of the existence of ideal measurements of the first kind on the structure of $\mathscr{P}$, we remember that quantum mechanics describes these consequences by the projection postulate according to which an ideal measurement of the first kind of the proposition $x$ (corresponding to the projection operator $P_{x}$ ) with positive result changes the state $W$ before the measurement into the state

$$
W_{+}=P_{x} W P_{x} / \operatorname{Tr}\left(W P_{x}\right)
$$

after the measurement. In particular, every ideal measurement of the first kind changes pure states into pure states, and the correspondence $W \rightarrow W_{+}$, given by (2.6), characterizes the proposition $x$ uniquely even if restricted to homogeneous states.

Accordingly, to every ideal measurement of the first kind there corresponds a one-to-one transformation in the set $\Pi(\mathscr{P})$ of all pure states which enables us to characterize these measurements also within the proposition calculus.

Definition 2. An ideal measurement of the first kind of the proposition $x \in \mathscr{P} \backslash\{\mathbf{0}, \mathbf{1}\}$ with positive result (IMFK) is a transformation $\tau_{x}$ in $\Pi(\mathscr{P})$ with the properties

(i) the domain $D\left(\tau_{x}\right)$ is equal to $\left\{S \in \Pi(\mathscr{P}) \mid x^{\prime} \notin S\right\}$,

(ii) $(\forall S) x \in \tau_{x}(S)$,

(iii) $(\forall z)(\forall S) \quad z \leftrightarrow x, \quad z \in S \Rightarrow z \in \tau_{x}(S)$,

where $x \leftrightarrow z$ denotes $x$ and $z$ are compatible and means that, in the frame of the proposition calculus, $x, x^{\prime}, z$ and $z^{\prime}$ generate a distributive sublattice of $\mathscr{P}$. The Eqs. (2.7) and (2.8) characterize measurements of the first kind with a positive outcome, and Eq. (2.9) requires the IMFK to be ideal.

Following Jauch and Piron, we postulate:

Axiom II. To all non-trivial propositions (i.e. to all $x \in \mathscr{P} \backslash\{\mathbf{0}, \mathbf{1}\}$ ) there exist at least one IMFK.

In [1] Jauch and Piron have asserted and - according to them proved that the Axioms I and II imply the covering law, i.e.

$(\forall x, b \in \mathscr{P})(\forall a \in \Omega(\mathscr{P})) \quad x \leqq b \leqq x \cup a \Rightarrow b=x \quad$ or $\quad b=x \cup a$. 
But this assertion is incorrect as the following example shows. Consider a lattice $\mathscr{V}$ with the diagram of Fig. 1.

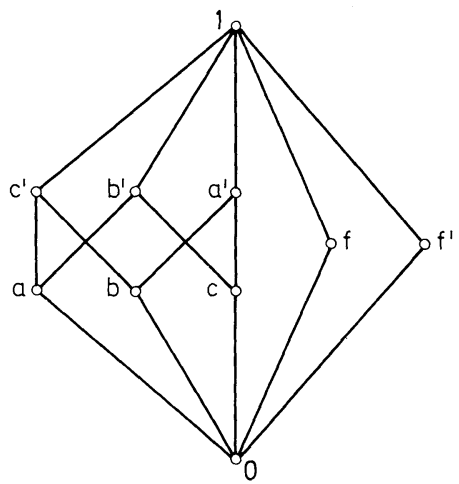

Fig. 1. By the definitions $\mathbf{0}^{\prime}=\mathbf{1}$ and $\left(x^{\prime}\right)^{\prime}=x$, the correspondance $x \rightarrow x^{\prime}$ becomes an orthocomplementation of $\mathscr{V}$

$\mathscr{V}$ has the atoms $a, b, c, f$ and $f^{\prime}$ and the prime filters $S_{a}=\left\{a, b^{\prime}, c^{\prime}, 1\right\}$, $S_{b}=\left\{a^{\prime}, b, c^{\prime}, 1\right\}, S_{c}=\left\{a^{\prime}, b^{\prime}, c, 1\right\}, S_{f}=\{f, 1\}$ and $S_{f^{\prime}}=\left\{f^{\prime}, 1\right\}$. Using the notations $A=\left\{a, b, c, a^{\prime}, b^{\prime}, c^{\prime}, f, f^{\prime}\right\}, B=\{a, b, c\}$ and $F=\left\{f, f^{\prime}\right\}$, we associate to every element $x \in A$ a transformation $\tau_{x}$ in $\Pi(\mathscr{V})$ with the properties

$(\alpha)$ the domain $D\left(\tau_{x}\right)$ of $\tau_{x}$ is equal to $\left\{S \in \Pi(\mathscr{V}) \mid x^{\prime} \notin S\right\}$,

$(\beta)(\forall x \in \Omega(\mathscr{V}))\left(\forall S \in D\left(\tau_{x}\right)\right) \quad \tau_{x}(S)=S_{x}$,

$(\gamma)(\forall x, y \notin F)\left(\forall S_{y} \in D\left(\tau_{x}\right)\right) \quad \tau_{x}\left(S_{y}\right)=S_{y}$,

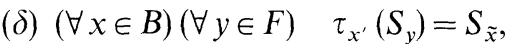

where $\tilde{x}$ denotes the successor of $x$ in the cycle $(a, b, c)$.

The following facts are easily checked:

Lemma 1. (a) $\mathscr{V}$ is a complete, atomic, orthomodular lattice.

(b) $\mathscr{V}$ does not satisfy the covering law.

(c) The conditions $(\alpha)$ to $(\delta)$ determine to each element $x \in A$ exactly one transformation $\tau_{x}$ in $\Pi(\mathscr{V})$, and these transformations have the properties (2.7) to (2.9).

Thus, if we interpret $\mathscr{V}$ as a proposition system then the above model satisfies the Axioms I and II but violates the covering law. This means that the assertion of Jauch and Piron cited above is incorrect and that their proof contains a gap ${ }^{3}$.

${ }^{3}$ The gap in the derivation of [1] lies in the unfounded deduction from $S_{a} \supset\left\{x \mid\left(e \cup a^{\prime}\right) \cap a \subset x\right\}$ to the relation $S_{a}=\left\{x \mid\left(e \cup a^{\prime}\right) \cap a \subset x\right\}$ which is needed for the proof (original notations from [1], p. 848). 


\section{$\S 3$}

In this section we will deduce the covering law from the Axioms I and II with the help of an additional assumption on ideal measurements of the first kind. For this purpose we quote at first some attributes of orthomodular lattices which are proved in the appendix or in [1].

Lemma 2. (a) In an atomic, orthomodular lattice $\mathscr{V}$, every element $\notin \Omega(\mathscr{V}) \cup\{\mathbf{0}\}$ is preceded by at least two different atoms.

(b) In an orthomodular lattice, the conjunction of (2.8) and (2.9) is equivalent to

$$
\left(x^{\prime} \cup y\right) \cap x \in \tau_{x}\left(S_{y}\right) .
$$

(c) In an orthomodular lattice $\mathscr{V}$, the covering law is equivalent to

$$
(\forall x \in \mathscr{V})(\forall a \in \Omega(\mathscr{V})) \quad\left(x^{\prime} \cup a\right) \cap x \in \Omega(\mathscr{V}) \cup\{\mathbf{0}\} .
$$

According to Lemma ( $2 \mathrm{~b}$ ), the properties of Definition 2 determine the atom corresponding to the state $\tau_{x}\left(S_{y}\right)$ after the measurement only up to the majorant $\left(x^{\prime} \cup y\right) \cap x$, where $\left(x^{\prime} \cup y\right) \cap x$ is different from $\mathbf{0}$ in the domain $D\left(\tau_{x}\right)$ of $\tau_{x}$. If this majorant is no atom, then it is, in view of Lemma ( $2 \mathrm{a})$, preceded by at least two atoms; hence there exist at least two different IMFKs associated to $x$. The covering law on the other hand prohibits, by Lemma $(2 \mathrm{c})$, that $\left(x^{\prime} \cup y\right) \cap x$ is preceded by two different atoms. These considerations lead directly to the additional postulate which must be imposed on a proposition system so that the covering law is valid.

Axiom III. The IMFK $\tau_{x}$ is uniquely determined by the properties (2.7) to (2.9).

This axiom means that the state resulting from an ideal measurement of the first kind on an object in a pure state depends only on the previous state and on the outcome of the measurement and has no general properties independent of the characteristics (2.7) to (2.9). This assumption conforms to the fact that in the Hilbert space formalism of quantum mechanics the projection postulate for pure states can be derived from the properties (2.7) to (2.9), and Axiom III is, in the authors opinion, physically meaningful and justified.

Theorem. In an atomic, orthomodular lattice the covering law is equivalent to the conjunction of the Axioms II and III.

Proof. 1) Let $\mathscr{V}$ be an atomic, orthomodular lattice satisfying the Axioms II and III. We consider two elements $z \in \mathscr{V}$ and $b \in \Omega(\mathscr{V})$ with the property

$$
\left(z^{\prime} \cup b\right) \cap z \notin \Omega(\mathscr{V}) \cup\{\mathbf{0}\} .
$$


By Lemma (2a) and Eq. (3.3), $\Omega(\mathscr{V})$ contains two different atoms $c_{1}, c_{2}$ with $c_{i} \geqq\left(z^{\prime} \cup b\right) \cap z$ and this implies, because of

$$
\left(z^{\prime} \cup y\right) \cap z=\mathbf{0} \Leftrightarrow z^{\prime} \geqq y \Leftrightarrow z^{\prime} \in S_{y},
$$

the existence of two different mappings

$$
\tau_{z}^{(i)}:\left\{S \in \Pi(\mathscr{V}) \mid z^{\prime} \notin S\right\} \mapsto \Pi(\mathscr{V}), \quad i=1,2
$$

with the properties

$$
\left(z^{\prime} \cup y\right) \cap z \in \tau_{z}^{(i)}\left(S_{y}\right) \quad \text { and } \quad \tau_{z}^{(i)}\left(S_{b}\right)=S_{c_{\imath}} .
$$

By Lemma (2b), these $\tau_{z}^{(i)}$ are IMFKs, which amounts to a contradiction to Axiom III. Thus the assumption (3.3) proves incompatible with our premises, and hence the covering law holds according to Lemma (2c).

2) Let $\mathscr{V}$ be an atomic, orthomodular lattice satisfying the covering law. We introduce the function $t$ by

$$
D(t)=\left\{(x, y) \in \mathscr{V} \times \Omega(\mathscr{V}) \mid x^{\prime} \geq y\right\} \quad \text { and } \quad t(x, y)=\left(x^{\prime} \cup y\right) \cap x .
$$

Owing to Eq. (3.4) and Lemma (2c), the range of $t$ is contained in $\Omega(\mathscr{V})$, and Lemma (2b) implies that the transformations $\tau_{x}$ in $\Pi(\mathscr{V})$, defined by $D\left(\tau_{x}\right)=\left\{S_{z} \mid(x, z) \in D(t)\right\}$ and $\tau_{x}\left(S_{y}\right):=S_{t(x, y)}$, are IMFKs. Now let $\kappa_{x}$ be an arbitrary transformation in $\Pi(\mathscr{V})$ with the properties (2.7) to (2.9). From Lemma (2b) it follows that

$$
(\forall(x, y) \in D(t)) \quad\left(x^{\prime} \cup y\right) \cap x \in \kappa_{x}\left(S_{y}\right),
$$

and Lemma (2c) yields

$$
(\forall(x, y) \in D(t)) \quad\left(x^{\prime} \cup y\right) \cap x \in \Omega(\mathscr{V}) .
$$

This implies $\tau_{x}=\kappa_{x}$ and hence the Axioms II and III are satisfied.

This theorem supplies a necessary and sufficient condition for the validity of the covering law in quantal proposition systems and thus closes the gap in the derivation of [1].

\section{Appendix}

Proof of Lemma (2a). All atomic, orthomodular lattices without any nontrivial element $\notin \Omega(\mathscr{V})$ have one of the diagrams of Fig. 2 and obviously satisfy Lemma (2a).

Let $\mathscr{V}$ be an atomic, orthomodular lattice with a nontrivial element $z$ exterior to $\Omega(\mathscr{V})$. Hence $\mathscr{V}$ includes a nonzero element $b$ with $b \leqq z$ so that the sublattice $V_{b z}$ generated by $b, b^{\prime}, z$ and $z^{\prime}$ is boolean. By $b \neq \mathbf{0}$, $z \neq 1$ and $b \leqq z, V_{b z}$ has the diagram of Fig. 3 and $z$ takes one of the 


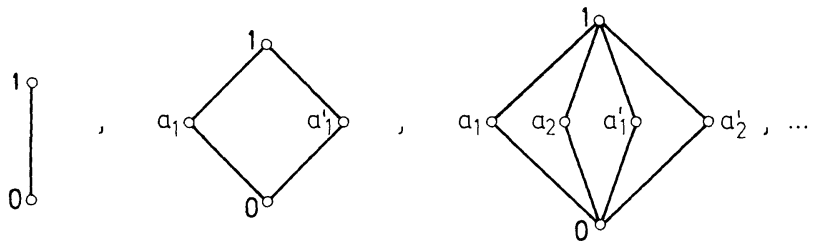

Fig. 2

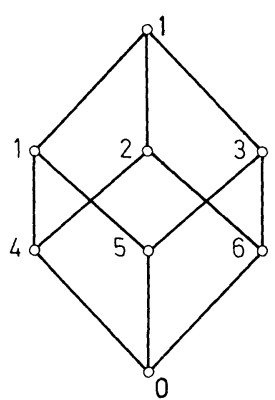

Fig. 3

places $1,2,3$. Without loss of generality let $z$ be equal to $x_{1}$ where we denote the element on place $n$ by $x_{n}$; then $z$ is preceded by $x_{4}$ and $x_{5}$. Because of $x_{4} \cap x_{5}=\mathbf{0}, x_{4}$ and $x_{5}$ are not preceded by a common atom and hence the atomicity of $\mathscr{V}$ implies that $z$ is preceded by at least two different atoms.

Proof of Lemma (2b). 1) Let $\mathscr{V}$ be an orthomodular lattice and let $\tau$ be a transformation in $\Pi(\mathscr{V})$ with the property

$$
(\exists x \in \mathscr{V} \backslash\{\mathbf{0}, \mathbf{1}\})\left(\forall y \in \Omega(\mathscr{V}), y \text { 主的) }\left(x^{\prime} \cup y\right) \cap x \in \tau\left(S_{y}\right) .\right.
$$

From $x \geqq x \cap\left(x^{\prime} \cup y\right)$ and the Eqs. (1), (2.2) we obtain

$$
(\forall y) \quad x \in \tau\left(S_{y}\right) .
$$

Next we consider two elements $k \in \mathscr{V}, y \in \Omega(\mathscr{V})$ with the properties $k \leftrightarrow x, y \geqq x^{\prime}$ and $k \geqq y$. From $k \leftrightarrow x$ it follows that

$$
\left(x^{\prime} \cup k\right) \cap x=\left(x^{\prime} \cap x\right) \cup(k \cap x)=k \cap x,
$$

and from $k \geqq y$ we get

$$
\left(x^{\prime} \cup k\right) \cap x \geqq\left(x^{\prime} \cup y\right) \cap x .
$$

Both equations together yield

$$
k \geqq k \cap x=\left(x^{\prime} \cup k\right) \cap x \geqq\left(x^{\prime} \cup y\right) \cap x,
$$

and owing to Eq. (1), this means $k \in \tau\left(S_{y}\right)$. Thus Eq. (3.1) implies the Eqs. (2.8) and (2.9). 
2) We now conversely assume that $\tau$ has the properties (2.8) and (2.9). From $x^{\prime} \leqq x^{\prime} \cup y$ it follows that $x^{\prime} \cup y \leftrightarrow x^{\prime}$ and $x^{\prime} \cup y \leftrightarrow x$, and from $y \leqq x^{\prime} \cup y$ we get $x^{\prime} \cup y \in S_{y}$; hence the assumption (2.9) yields

$$
x^{\prime} \cup y \in \tau\left(S_{y}\right) .
$$

From (2), (2.8) and (2.4) we finally arrive at $\left(x^{\prime} \cup y\right) \cap x \in \tau\left(S_{y}\right)$ which proves the second half of Lemma $(2 b)$.

Lemma $(2 \mathrm{c})$ is proved in [1].

\section{References}

1. Jauch, J. M., Piron, C.: Helv. Phys. Acta 42, 842 (1969).

2. - Foundations of Quantum Mechanics. Reading, Mass.: Addison-Wesley 1968.

3. Holland, S. S.jr.: The current interest in orthomodular lattices. In: Abbott, J.C. (Ed.): Trends in Lattice Theory. New York: van Nostrand, Reinhold Co. 1970.

W. Ochs

Sektion Physik der Universität

Lehrstuhl Prof. Süßmann

D-8000 München 13

Schellingstr. 2-8

Germany 